\title{
Timing of Quiescence and Uveitis Recurrences After Cataract Surgery in Patients with a History of Uveitis
}

\author{
Austin Rohl · Jennifer L. Patnaik · D. Claire Miller · Anne M. Lynch • \\ Alan G. Palestine (D
}

Received: May 18, 2021 / Accepted: June 10, 2021 / Published online: June 23, 2021

(c) The Author(s) 2021

\begin{abstract}
Introduction: In patients with a history of uveitis, a 60-90-day period of quiescence prior to cataract surgery has been historically recommended. These recommendations were based mostly on expert opinion. The purpose of this study was to further examine and understand uveitis quiescence timing and outcomes of eyes that do have a recurrence of uveitis after cataract surgery.

Methods: Patients with a history of uveitis undergoing cataract surgery at the University of Colorado Sue-Anschutz Rodgers Eye Center from 2014 to 2018 were studied. Main outcome measures were best corrected visual acuity (BCVA) at 6 months, intraocular pressure (IOP) at 6 months, and recurrences of uveitis within 90 days of surgery. Timing of cataract surgery in relation to preoperative uveitis quiescence was recorded.
\end{abstract}

Supplementary Information The online version contains supplementary material available at https:// doi.org/10.1007/s40123-021-00363-0.

A. Rohl · J. L. Patnaik · D. Claire Miller .

A. M. Lynch · A. G. Palestine $(\varangle)$

Department of Ophthalmology, University of

Colorado School of Medicine, 1675 Aurora Ct, F731,

Aurora, CO 80045, USA

e-mail: Alan.palestine@cuanschutz.edu
Results: A total of 149 eyes from 114 patients were included. Uveitis recurrence was seen in 31 $(20.8 \%)$ of the 149 eyes with a history of uveitis undergoing phacoemulsification. The mean logMAR change or improvement in vision was 0.429 in eyes with a recurrence of uveitis, and 0.318 in eyes without, a difference that was not statistically significant $(p=0.292)$. Eyes with a recurrence of uveitis displayed higher IOP at 6 months compared to eyes without a recurrence, at 16.1 versus $13.0 \mathrm{mmHg}$ respectively $(p=0.0009)$. Eyes that were quiescent for 30 days or less were more likely to have a uveitis recurrence (OR 6.11, 95\% CI 2.48-15.06, $p=0.0001)$.

Conclusions: Our study shows that a quiescence period of at least 30 days is beneficial in reducing uveitis recurrence after cataract surgery. It is unclear what the long-term consequences of a uveitis recurrence after cataract surgery are, though it appears there may be elevated IOP in patients with recurrences, and thus patients with a history of uveitis should be counseled carefully about this potential risk.

Keywords: Cataract surgery; Uveitis; Uveitis recurrence 


\section{Key Summary Points}

\section{Why carry out this study?}

Eyes with uveitis often develop cataracts, and there is an increased risk of the uveitis recurring after cataract surgery.

To examine the outcomes of eyes that have a recurrence of uveitis after cataract surgery and the risk factors surrounding those recurrences, with a focus on timing of quiescence prior to surgery.

\section{What was learned from the study?}

Eyes with active inflammation or inflammation within 30 days were at significantly higher risk for uveitis recurrence (OR 6.11, 95\% CI 2.48-15.06, $p=0.0001)$.

Eyes with uveitis recurrences had similar visual acuity gains from cataract surgery as those without recurrences, but intraocular pressure at 6 months was higher in eyes with a recurrence.

\section{DIGITAL FEATURES}

This article is published with digital features, including a summary slide, to facilitate understanding of the article. To view digital features for this article, go to https://doi.org/10.6084/ m9.figshare.14755308.

\section{INTRODUCTION}

One of the most common complications of uveitis is cataract formation, whether due to inflammation or the corticosteroid therapy that uveitic eyes may receive. Cataract surgery in uveitic eyes presents unique challenges. Surgeons may encounter poor dilation, posterior synechiae, iris atrophy, anterior segment bleeding, elevated intraocular pressure (IOP), cystoid macular edema (CME), and

postoperative inflammation and uveitis recurrence [1]. Careful surgical planning and preoperative management is crucial to the success of cataract surgery outcomes in these eyes.

Historically, experts have recommended anywhere from a 60- to 90-day or more period of uveitic quiescence prior to cataract surgery in order to prevent surgically induced uveitis recurrences [2]. However, these recommendations are mostly based on expert opinion, and there are few studies specifically looking at these timing recommendations and outcomes of patients who do have uveitis recurrences.

The purpose of this retrospective study was to examine a relatively large cohort of patients with a history of uveitis who underwent cataract surgery, and attempt to identify risk factors, characteristics, and outcomes of the eyes that do have uveitis recurrences, with a specific focus on timing of quiescence prior to surgery. Our hope is that we can better understand the timing of cataract surgery in uveitic eyes and counsel patients more confidently regarding outcomes and risk of uveitis recurrence.

\section{METHODS}

A retrospective cohort study in patients with uveitis was conducted using records from a cataract outcomes registry developed by the Department of Ophthalmology at the University of Colorado School of Medicine. The study prospectively received approval from the Colorado Multiple Institutional Review Board (COMIRB Protocol 18-0122), and all research conformed to the tenets of the Declaration of Helsinki. In a manner similar to our previous publications [3], medical charts were reviewed, and data were entered into Research Electronic Data Capture (REDCap; Vanderbilt University, Nashville, Tennessee) database, a secure, webbased application. The registry includes patient demographic information, medical history, preoperative medication history, intraoperative and postoperative complications, and preoperative and postoperative eye examinations.

This study included patients with a documented history of uveitis who underwent uncomplicated phacoemulsification cataract 
surgery with intraocular lens implantation, with or without combined procedures (e.g., pars plana vitrectomy, minimally invasive glaucoma surgery, glaucoma drainage device insertion, corneal surgery) between January 1, 2014, and December 31, 2018, at the University of Colorado Sue-Anschutz Rodgers Eye Center. Patients were followed for at least 6 months after surgery. Exclusion criteria included eyes with a history of uveitis only in the non-operative eye, complicated cataract surgery (defined as posterior capsular tear, vitreous loss, iris trauma, retained lens material, and zonular dialysis), and eyes with a history of episcleritis, scleritis, or keratitis but without anterior uveitis or other intraocular inflammation.

Cataract surgeries were performed using phacoemulsification with clear corneal incisions. Brand moxifloxacin was either injected intracamerally at the end of surgery or placed topically through a soaked collagen shield. Following surgery, patients were prescribed a topical postoperative eyedrop regimen consisting of a fluoroquinolone antibiotic, nonsteroidal anti-inflammatory drugs, and corticosteroids. The antibiotic was typically discontinued at the week 1 postoperative appointment. Depending on surgeon preference, the steroid was prescribed as either one drop twice daily for 2 weeks (using difluprednate $0.05 \%)$, or four times daily for 1 week, followed by a weekly taper for 3 weeks (using prednisolone acetate 1\%). Pre- and perioperative steroids for uveitis control were prescribed at the discretion of the treating physician. In eyes with known infectious viral uveitis etiologies, antiviral therapy was either maintained or increased preoperatively at the discretion of the treating physician.

Visual acuity and IOP were determined by a trained, certified ophthalmic technician using a Snellen chart at distance viewing and Goldmann applanation tonometry, respectively. The presence and grading of the anterior chamber cell was determined by the physician using the SUN Working Group grading scheme for anterior chamber (AC) cells [4]. Macular optical coherence tomography imaging and $\mathrm{CME}$ diagnosis was made at the discretion of the treating physician. Uveitis activity status was defined as quiet if there was less than $0.5+\mathrm{AC}$ cell or if the treating physician documented so in cases of more posterior disease. Charts were reviewed manually to determine when the most recent flare of uveitis began and ended to analyze quiescence timing in relation to the date of cataract surgery.

Recurrence of uveitis after cataract surgery was defined as any anterior chamber cell, vitreous cell, or CME postoperatively within a 90-day period from the time of surgery necessitating addition or escalation of steroid therapy or immunosuppressive agents. This 90-day period was arbitrarily chosen, as we believe that inflammation occurring only after 90 days would be difficult to attribute to surgery. This definition is similar to those used by Elgohary et al. [5] and Sharief et al. [6]. With the exception of an eye requiring an urgent glaucoma tube surgery and an eye that underwent goniosynechiolysis at the time of a planned vitreous biopsy, no other eyes underwent surgery in the 90-day postoperative period. Patients with no documented history of CME prior to cataract surgery, who only had new CME postoperatively without the presence of persistent anterior chamber cell or vitreous cell as well were not considered to have recurrent uveitis and were considered to have pseudophakic CME (Irvine-Gass syndrome).

Basic frequencies were used to describe categorical variables. Continuous variables were described as means, standard deviations, and medians. Comparisons of eye characteristics were performed using logistic regressions with general estimating equations (GENMOD; SAS, Inc., Cary, North Carolina) to account for the intrasubject correlation for patients who had both eyes included in the analyses. Odds ratios and 95\% confidence intervals (CIs) were used for measures of association. $p$ values less than 0.05 were considered statistically significant. Statistical analysis was performed using SAS version 9.4 software (Cary, North Carolina).

\section{RESULTS}

There were 10,758 eyes in the Cataract Outcome Registry between 2014 and 2018. A total 
Table 1 Characteristics of patients and eyes with a history of uveitis prior to cataract surgery

\begin{tabular}{ll}
\hline & $n(\%)$ \\
\hline Patients & 114 \\
Age (years), mean (SD) & $55.8(16.1)$ \\
Gender & \\
Male & $40(35.1 \%)$ \\
Female & $74(64.9 \%)$ \\
Race/ethnicity & \\
White, non-Hispanic & $72(63.2 \%)$ \\
Hispanic & $9(7.9 \%)$ \\
African-American & $24(21.0 \%)$ \\
Asian/Native Hawaiian/PI & $4(3.5 \%)$ \\
Other & $2(1.8 \%)$ \\
Unknown & $3(2.6 \%)$ \\
Patient with 2 eyes included in study & $35(30.7 \%)$ \\
Eyes & 149 \\
Uveitis classification & \\
Anterior & $79(53.0 \%)$ \\
Intermediate & $29(19.5 \%)$ \\
Panuveitis & $25(16.8 \%)$ \\
Posterior & $15(10.1 \%)$ \\
Unspecified & $1(0.7 \%)$ \\
Time since uveitis diagnosis, days & \\
Mean (SD) & $2777(3955)$ \\
Median & 1441 \\
Range & $15-19,816$ \\
Uveitis status at time of surgery & \\
Active & \\
\hline
\end{tabular}

of 288 eyes were categorized in the database as having a history of uveitis. Almost half or 139 eyes were excluded using the criteria described. A total of 149 eyes from 114 patients were
Table 2 Recurrence of uveitis after cataract surgery by time quiescence

\begin{tabular}{|c|c|c|}
\hline & $\begin{array}{l}\text { Recurrence of uveitis } \\
n / N(\%)\end{array}$ & $p$ value \\
\hline Total & $31 / 149(20.8 \%)$ & - \\
\hline \multicolumn{3}{|l|}{ Days quiet } \\
\hline Active & $8 / 18(44.4 \%)$ & \\
\hline$<14$ days & $4 / 7(57.1 \%)$ & \\
\hline $14-30$ days & $2 / 3(66.7 \%)$ & \\
\hline $30-60$ days & $1 / 11(9.1 \%)$ & \\
\hline $60-90$ days & $2 / 15(13.3 \%)$ & \\
\hline 90-180 days & $4 / 21(19.0 \%)$ & \\
\hline$>180$ days & $10 / 74(13.5 \%)$ & \\
\hline \multicolumn{3}{|l|}{ Days quiet } \\
\hline Active or $<30$ days & $14 / 28(50.0 \%)$ & \\
\hline$\geq 30$ days & $17 / 121(14.0 \%)$ & 0.0004 \\
\hline \multicolumn{3}{|l|}{ Days quiet } \\
\hline Active or $<60$ days & $15 / 39(38.5 \%)$ & \\
\hline$\geq 60$ days & $16 / 110(14.6 \%)$ & 0.004 \\
\hline \multicolumn{3}{|l|}{ Days quiet } \\
\hline Active or $<90$ days & $17 / 54(31.5 \%)$ & \\
\hline$\geq 90$ days & $14 / 95(14.7 \%)$ & 0.021 \\
\hline \multicolumn{3}{|l|}{ Days quiet } \\
\hline Active or $<180$ days & $21 / 75(28.0 \%)$ & \\
\hline$\geq 180$ days & $10 / 74(13.5 \%)$ & 0.038 \\
\hline
\end{tabular}

included in this study. The demographics of patients and characteristics of the eyes are summarized in Table 1. Uveitis diagnosis and associations are provided in Supplemental Table S1. A total of 44 eyes underwent combined cataract surgery, 10 with vitrectomy, 31 with glaucoma surgery, and 3 with corneal surgery.

Recurrences of uveitis and quiescence timing for such eyes are shown in Table 2 . The overall incidence of recurrence of uveitis after cataract surgery was $20.8 \%$ (31 out of 149 eyes). Eyes 
with active inflammation or inflammation within 30 days were at significantly higher risk for uveitis recurrence (OR 6.11, 95\% CI $2.48-15.06, p=0.0001)$. When comparing eyes that were either actively inflamed or quiet for less than 30 days to eyes quiet more or equal to 30 days, there was a statistically significant higher rate of recurrence of uveitis in the former, with a rate of $50 \%$ versus $14 \%(p=0.0004)$. Differences were also significant with a 60-day cutoff, 90-day cutoff, and 180-day cutoff. There were high recurrence rates prior to 30 days of quiescence, while the longer time period of quiescence had similar lower recurrence rates.

Table 3 shows the characteristics of the recurrent uveitis analyzed by quiescence, uveitis classification, and treatment modality for the recurrence. In total, 16 eyes with recurrence were treated with just topical steroids, 9 with sub-tenon's Kenalog (STK) injection, and 6 with Ozurdex (® (dexamethasone intravitreal implant, Allergan Inc, Irvine, CA) injection, one of those receiving both Ozurdex and orally administered prednisone.

Eyes with intermediate uveitis, patients on one or two disease-modifying anti-rheumatic drugs (DMARDs), eyes that underwent combined cataract surgery, and eyes with a history of more than one episode of uveitis all had notably higher rates of uveitis recurrence, though not statistically significantly, as noted in Table 4. Only patients with a history of CME prior to cataract surgery had a statistically significant higher rate of recurrence of uveitis after cataract surgery $(p=0.013)$. Of note, among the 31 eyes with recurrences, only two of those eyes had a history of a single uveitis episode, all others had more than one historic episode.

Eyes not given additional perioperative steroids or only given additional steroid eye drops tended to have a more distant uveitis flare, with a median time since last episode of 474 days for the group given no additional perioperative steroids versus 49 days in the group that was given systemic steroids and STK or intravitreal steroids.

For eyes that experienced a recurrence of uveitis, both preoperative and 6-month postoperative best-corrected visual acuity (BCVA) was worse than eyes that did not experience a recurrence of uveitis, though this difference was not significant. The mean logMAR change or improvement in vision was 0.429 in those eyes with a recurrence of uveitis, and 0.318 in eyes without, a difference that was also not significantly different $(p=0.292)$.

Preoperative mean IOP was similar between eyes that had a recurrence of uveitis and eyes without, 16.5 and $15.1 \mathrm{mmHg}$, respectively $(p=0.302)$. IOP at $1-3$ months post cataract surgery was also similar between the two groups, at 13.2 compared to $13.4 \mathrm{mmHg}$

Table 3 Uveitis recurrences by quiescence category, uveitis classification, and treatment modality of recurrences

\begin{tabular}{lllllllll}
\hline Days quiet & Anterior & Intermediate & Posterior & Panuveitis & $\begin{array}{l}\text { Total number } \\
\text { of recurrences }\end{array}$ & $\begin{array}{l}\text { Treated with } \\
\text { topical } \\
\text { steroids }\end{array}$ & $\begin{array}{l}\text { Treated } \\
\text { with } \\
\text { STK }\end{array}$ & $\begin{array}{l}\text { Treated } \\
\text { with } \\
\text { Ozurdex }\end{array}$ \\
\hline Active & 1 & 3 & 0 & 4 & 8 & 1 & 4 & 3 \\
$<14$ days & 1 & 1 & 1 & 1 & 4 & 3 & 1 & 0 \\
$14-30$ days & 0 & 2 & 0 & 0 & 2 & 2 & 0 & 0 \\
$30-60$ days & 0 & 0 & 0 & 1 & 1 & 0 & 1 & 0 \\
$60-90$ days & 1 & 0 & 0 & 1 & 2 & 1 & 0 & 1 \\
$90-180$ days & 1 & 1 & 1 & 1 & 4 & & 2 & 1 \\
$>180$ days & 8 & 1 & 0 & 1 & 10 & 8 & 1 & 1 \\
\hline
\end{tabular}


Table 4 Rates of recurrence of uveitis by demographic and clinical characteristics

\begin{tabular}{|c|c|c|c|}
\hline & Recurrence of uveitis & Did not have recurrence & $p$ value \\
\hline \multicolumn{4}{|l|}{ Gender } \\
\hline Male & $12 / 46(26.1 \%)$ & $34 / 46(73.9 \%)$ & \\
\hline Female & $19 / 103(18.4 \%)$ & $84 / 103(81.6 \%)$ & 0.308 \\
\hline \multicolumn{4}{|l|}{ Race/ethnicity } \\
\hline White & $20 / 92(21.7 \%)$ & $72 / 92(78.3 \%)$ & Reference \\
\hline Black & $9 / 34(26.5 \%)$ & $25 / 34(73.5 \%)$ & 0.574 \\
\hline Other & $2 / 23(8.7 \%)$ & $21 / 23(91.3 \%)$ & 0.181 \\
\hline \multicolumn{4}{|l|}{ Uveitis type } \\
\hline Anterior & $13 / 79(16.5 \%)$ & $66 / 79(83.5 \%)$ & Reference \\
\hline Intermediate & $10 / 29(34.5 \%)$ & $19 / 29(65.5 \%)$ & 0.054 \\
\hline Panuveitis & $6 / 25(24.0 \%)$ & $19 / 25(76.0 \%)$ & 0.396 \\
\hline Posterior & $2 / 15(13.3 \%)$ & $13 / 15(86.7 \%)$ & 0.772 \\
\hline Unspecified & $0 / 1(0 \%)$ & $1 / 1(100 \%)$ & - \\
\hline Length of diagnosis of uveitis, median (range) & $1355(15-8365)$ & $1502(73-19,816)$ & 0.480 \\
\hline \multicolumn{4}{|l|}{ DMARD } \\
\hline 0 & $21 / 122(17.2 \%)$ & $101 / 122(82.8 \%)$ & Reference \\
\hline 1 & $7 / 20(35.0 \%)$ & $13 / 20(65.0 \%)$ & 0.075 \\
\hline 2 & $3 / 7(42.9 \%)$ & $4 / 7(57.1 \%)$ & 0.099 \\
\hline \multicolumn{4}{|l|}{ Combined procedure } \\
\hline Yes & $13 / 44(29.6 \%)$ & $31 / 44(70.4 \%)$ & \\
\hline No & $18 / 105(17.1 \%)$ & $87 / 105(82.9 \%)$ & 0.096 \\
\hline \multicolumn{4}{|l|}{ Previous CME } \\
\hline Yes & $14 / 39(35.9 \%)$ & $25 / 39(64.1 \%)$ & \\
\hline No & $17 / 109(15.6 \%)$ & $92 / 109(84.4 \%)$ & 0.013 \\
\hline \multicolumn{4}{|l|}{ History of $>1$ episode uveitis } \\
\hline Yes & $29 / 119(24.4 \%)$ & $90 / 119(75.6 \%)$ & \\
\hline No & $2 / 27(7.4 \%)$ & $25 / 27(92.6 \%)$ & 0.073 \\
\hline CDE, median (range) & $2.97(0-14.52)$ & $3.45(0-17.93)$ & 0.780 \\
\hline \multicolumn{4}{|l|}{ Length of surgery (min) } \\
\hline Median (range) & $21(9-130)$ & $16(7-139)$ & 0.318 \\
\hline \multicolumn{4}{|l|}{ Preoperative uveitis treatment plan } \\
\hline No perioperative steroids & $5 / 58(8.6 \%)$ & $53 / 58(91.4 \%)$ & - \\
\hline Only topical drops & $5 / 14(35.7 \%)$ & $9 / 14(64.3 \%)$ & - \\
\hline
\end{tabular}


Table 4 continued

\begin{tabular}{|c|c|c|c|}
\hline & Recurrence of uveitis & Did not have recurrence & $p$ value \\
\hline Systemic steroids (IV or oral) ${ }^{a}$ & $10 / 37(27.0 \%)$ & $27 / 37(73.0 \%)$ & - \\
\hline STK or intravitreal steroids ${ }^{\mathrm{a}}$ & $4 / 17(23.5 \%)$ & $13 / 17(76.5 \%)$ & - \\
\hline Systemic steroids AND STK or intravitreal steroids ${ }^{a}$ & $7 / 23(30.4 \%)$ & $16 / 23(69.6 \%)$ & - \\
\hline \multicolumn{4}{|l|}{ Preoperative IOP } \\
\hline$n$ & 31 & 116 & \\
\hline Mean (SD) & $16.5(7.8)$ & $15.1(5.6)$ & \\
\hline Median (range) & $15.0(5-46)$ & $14.0(4-42)$ & 0.302 \\
\hline \multicolumn{4}{|l|}{ IOP $1-3$ months } \\
\hline$n$ & 30 & 107 & \\
\hline Mean (SD) & $13.2(3.4)$ & $13.4(3.8)$ & 0.693 \\
\hline Median (range) & $12.5(8-22)$ & $13.0(4-30)$ & \\
\hline \multicolumn{4}{|l|}{ IOP $4-6$ months } \\
\hline$n$ & 30 & 104 & \\
\hline Mean (SD) & $16.1(6.4)$ & $13.0(3.3)$ & 0.0009 \\
\hline Median (range) & $14.5(6-33)$ & $13.0(5-22)$ & \\
\hline \multicolumn{4}{|l|}{ logMAR preoperative } \\
\hline Mean (SD) & $0.796(0.73)$ & $0.555(0.69)$ & \\
\hline Median (range) & $0.398(0-3)$ & $0.301(0-3)$ & 0.113 \\
\hline \multicolumn{4}{|l|}{$\log$ MAR 6 month follow-up } \\
\hline Mean (SD) & $0.367(0.39)$ & $0.237(0.44)$ & \\
\hline Median (range) & $0.176(-0.125$ to 1.398$)$ & $0.097(-0.125-2.301)$ & 0.144 \\
\hline \multicolumn{4}{|l|}{ logMAR change } \\
\hline Mean (SD) & $0.429(0.53)$ & $0.318(0.50)$ & \\
\hline Median (range) & 0.301 & 0.176 & 0.292 \\
\hline
\end{tabular}

${ }^{\text {a }}$ With or without additional topical steroids

$(p=0.693)$, but at 4-6 months eyes with a recurrence of uveitis displayed higher IOP compared to eyes without a recurrence, 16.1 versus $13.0 \mathrm{mmHg}$ respectively, and this difference was statistically significant $(p=0.0009)$.

Of the 18 eyes deemed active at the time of cataract surgery, three eyes underwent combination surgery due to the need for urgent glaucoma valve surgery, two for angle closure glaucoma, and two underwent combination surgery due to need for a Retisert ${ }^{\circledR}$ (fluocinolone acetonide intravitreal implant, Bausch and Lomb, Rochester, NY) implant. Only one patient with a recurrence of uveitis developed significant IOP elevations requiring glaucoma surgery within 90 days. One patient with a recurrence of uveitis had a preoperative diagnosis of iris bombe and subsequently developed 
recurrent posterior synechiae within 3 months after cataract surgery and required goniosynechialysis at the time of a planned vitreous biopsy for further diagnosis of their disease process. No patients had recurrence of herpetic eye disease (interstitial keratitis, endotheliitis, etc.).

\section{DISCUSSION}

Expert consensus opinion has long held that cataract surgery in patients with uveitis should be delayed until the eye is quiet or free of inflammation for at least 60-90 days in order to prevent postoperative uveitis recurrence $[2,7,8]$. Our findings confirm that eyes with active inflammation or inflammation within 30 days were at significantly higher risk (estimated to be sixfold greater odds) for postoperative rebound inflammation. These quiescence timing cutoffs become less clear at 60 days and beyond in our study.

Few prior studies have looked at quiescence timing in detail, in part due to most studies excluding eyes if they were not quiescent for 90 days or more. A study by Takai et al. included eyes that were active within 90 days, and concluded that uveitis recurrence occurred more often when uveitis was quiet for less than 3 months preoperatively, with a $43.1 \%$ recurrence rate, versus cases quiet for more than 3 months, a 20.7\% recurrence rate [9] .Their study did not specify the definition of a uveitis recurrence, and they were not specific in the timing of the aforementioned recurrences, as they mentioned only a $13.4 \%$ recurrence rate within the first 3 months postoperatively [9]. Ji et al. examined 408 eyes with a history of Vogt-Koyanagi-Harada disease and compared those quiet at least 3 months and those with quiescence for only 1-3 months and found no difference in BCVA or postoperative complications including uveitis recurrence rate [10]. These findings may not be applicable to other types of uveitis, however. Bélair et al. demonstrated that eyes with uveitis undergoing cataract surgery with active inflammation within 3 months before surgery portended an increased risk of CME when compared to eyes without inflammation in that period [11]

Previous studies have established that cataract surgery in patients with uveitis is generally safe and associated with good visual acuity outcomes, with around $70 \%$ of patients achieving $20 / 40$ or better in a meta-analysis by Mehta et al. [8]. We found in our study that while both pre- and postoperative BCVA were better in eyes without recurrences, these differences were not statistically significant, and that the gain in BCVA was similar between eyes with and eyes without uveitis recurrence. This is similar to findings by Liu et al., which demonstrated that 6-month visual acuity outcomes were not compromised in the 91 eyes with uveitis recurrences [12]. Similarly, Kawaguchi et al. found no differences in visual acuity outcomes at 6 months in 17 eyes with uveitis that experienced a recurrence [13]. However, Abbouda et al. found that eyes with what they defined as having more than one "postoperative relapse year" had a trend towards worse visual acuity outcomes, though this difference was not statistically significant [14]. Ozates et al. found uveitis relapse rates actually decreased overall postoperatively in a group of 105 eyes, though not commenting or differentiating visual acuity outcomes from those that did recur early versus those that did not [15]. In addition, Reddy et al. showed that among eyes without prior uveitis, 3 -month postoperative visual acuity outcomes were no different in eyes that had persistent anterior uveitis after surgery versus those that did not [3].

Our study shows that IOP at 4-6 months post surgery in eyes with a uveitis recurrence was higher than those without recurrence. No previous studies have compared IOP outcomes in eyes with uveitis recurrences versus eyes without recurrences after cataract surgery. In fact, few studies have looked at IOP outcomes in eyes with uveitis undergoing cataract surgery. Balta et al. found that 9 of 55 eyes with uveitis that underwent cataract surgery had elevated IOP [16]. Mora et al. studied 52 eyes, and although average 2-week postoperative IOP was elevated, IOP at final follow-up was not significantly different than baseline [17]. Ram et al. included 108 eyes, of which three developed 
postoperative glaucoma and optic atrophy with final visual acuity of count fingers [18]. Reddy et al. showed that eyes with persistent anterior uveitis (PAU) after cataract surgery had a mean IOP $1.1 \mathrm{mmHg}$ higher than those eyes without PAU [3]. We postulate that eyes that experience uveitis recurrences endure higher IOP in that period, either related to a steroid effect on IOP [19] or an innate propensity for higher pressures related to uveitis recurrence.

The principal limitation of this study is its retrospective nature. Specifically, variability in postoperative drop regimens, follow-up intervals, uveitis treatment regimens, and diagnostic testing for CME. The retrospective nature of this study, however, did allow for a relatively larger sample size, one which would be difficult to obtain in a prospective study. In terms of visual acuity outcomes, the conversion to logMAR allowed for comparison by making the data analyzable, but our study was likely underpowered to detect small significant differences. However, the fact that we could not detect a difference provides a baseline for power calculations in future larger studies to attempt to detect differences related to uveitis recurrences. It is difficult to discern from our analysis whether eyes with frequent recurrences are inherently more prone to uveitis recurrence, or if the duration of quiescence is truly important to preventing uveitis recurrences postoperatively.

\section{CONCLUSION}

Our study indicates that in eyes with a history of uveitis, a preoperative period of quiescence of at least 30 days is beneficial in preventing postoperative uveitis recurrence after cataract surgery. It is unclear what the consequences of a uveitis recurrence are, as visual acuity gains in our study were the same among eyes with uveitis recurrences and eyes without, and final visual acuity outcomes were not statistically different between the groups. IOP is higher in eyes that have a uveitis recurrence, though the long-term impact of this is unknown and needs further investigation. At least a 30-day period of quiescence, influenced by other surgical considerations, and counseling all patients on the possibility of uveitis recurrence after cataract surgery are reasonable.

\section{ACKNOWLEDGEMENTS}

Funding. This research was supported in part by an unrestricted grant to the Department of Ophthalmology at the University of Colorado School of Medicine from Research to Prevent Blindness, New York. Funding was provided for cataract surgery outcomes database maintenance, data abstraction, and statistical analysis. No funding or sponsorship was received for publication of this article.

Authorship. All named authors meet the International Committee of Medical Journal Editors (ICMJE) criteria for authorship for this article, take responsibility for the integrity of the work as a whole, and have given their approval for this version to be published.

Authorship Contributions. Conceptualization: Austin J. Rohl and Alan G. Palestine; Methodology: Austin J. Rohl, Alan G. Palestine, D. Claire Miller, Jennifer L. Patnaik, Anne M. Lynch; Statistical Analysis: Jennifer L. Patnaik; Manuscript drafting: Austin J. Rohl and Alan G. Palestine; Manuscript review and editing: Alan G. Palestine, D. Claire Miller, Jennifer L. Patnaik, Anne M. Lynch.

Disclosures. Austin J. Rohl, Jennifer L. Patnaik, D. Claire Miller, Anne M. Lynch, and Alan G. Palestine have nothing to disclose.

Compliance with Ethics Guidelines. The study prospectively received approval from the Colorado Multiple Institutional Review Board (COMIRB Protocol 18-0122), and all research conformed to the tenets of the Declaration of Helsinki.

Data Availability. The datasets generated during and/or analyzed during the current study are available from the corresponding author on reasonable request. 
Open Access. This article is licensed under a Creative Commons Attribution-NonCommercial 4.0 International License, which permits any non-commercial use, sharing, adaptation, distribution and reproduction in any medium or format, as long as you give appropriate credit to the original author(s) and the source, provide a link to the Creative Commons licence, and indicate if changes were made. The images or other third party material in this article are included in the article's Creative Commons licence, unless indicated otherwise in a credit line to the material. If material is not included in the article's Creative Commons licence and your intended use is not permitted by statutory regulation or exceeds the permitted use, you will need to obtain permission directly from the copyright holder. To view a copy of this licence, visit http://creativecommons.org/licenses/by$\mathrm{nc} / 4.0 /$.

\section{REFERENCES}

1. Van Gelder RN, Leveque TK. Cataract surgery in the setting of uveitis. Curr Opin Ophthalmol. 2009;20(1):42.

2. Rojas B, Foster CS. Cataract surgery in patients with uveitis. Curr Opin Ophthalmol. 1996;7(1):11-6.

3. Reddy AK, Patnaik JL, Miller DC, Lynch AM, Palestine AG, Pantcheva MB. Risk factors associated with persistent anterior uveitis after cataract surgery. Am J Ophthalmol. 2019;206:82-6.

4. Standardization of Uveitis Nomenclature (SUN) Working Group. Standardization of uveitis nomenclature for reporting clinical data. Results of the First International Workshop. Am J Ophthalmol. 2005;140(3):509-16.

5. Elgohary MA, McCluskey PJ, Towler HM, et al. Outcome of phacoemulsification in patients with uveitis. Br J Ophthalmol. 2007;91(7):916-21.

6. Sharief L, Lightman S, Baltinas J, Tomkins-Netzer O. Long-term effect of cataract phacoemulsification on the inflammation control and clinical outcome in uveitis patients. Clin Exp Ophthalmol. 2018;46(9): 1048-54.

7. Jancevski M, Foster CS. Cataracts and uveitis. Curr Opin Ophthalmol. 2010;21(1):10-4.
8. Mehta S, Linton MM, Kempen JH. Outcomes of cataract surgery in patients with uveitis: a systematic review and meta-analysis. Am J Ophthalmol. 2014;158(4):676-92.

9. Takai N, Kobayashi T, Kida T, Ikeda T. Clinical features of Japanese patients with ocular inflammation and their surgical procedures over the course of 20 years. Clin Ophthalmol. 2020;14:2799.

10. Ji Y, Hu K, Li C, et al. Outcome and prognostic factors of phacoemulsification cataract surgery in Vogt-Koyanagi-Harada uveitis. Am J Ophthalmol. 2018;196:121-8.

11. Bélair ML, Kim SJ, Thorne JE, et al. Incidence of cystoid macular edema after cataract surgery in patients with and without uveitis using optical coherence tomography. Am J Ophthalmol. 2009;148(1):128-35.

12. Liu $\mathrm{X}, \mathrm{Zhao} \mathrm{C}, \mathrm{Xu} \mathrm{T}$, et al. Visual prognosis and associated factors of phacoemulsification and intraocular lens implantation in different uveitis entities in Han Chinese. Ocul Immunol Inflamm. 2017;25(3):349-55.

13. Kawaguchi T, Mochizuki M, Miyata K, Miyata N. Phacoemulsification cataract extraction and intraocular lens implantation in patients with uveitis. J Cataract Refract Surg. 2007;33(2):305-9.

14. Abbouda A, Tortorella P, Restivo L, Santoro E, De Marco F, La Cava M. Follow-up study of over three years of patients with uveitis after cataract phacoemulsification: outcomes and complications. Semin Ophthalmol. 2016;31(6):532-41.

15. Ozates S, Berker N, Ozdal PC, Erol YO. Phacoemulsification in patients with uveitis: long-term outcomes. BMC Ophthalmol. 2020;20(1):1-8.

16. Balta O, Sungur G, Acar MA, Kosker M, Yakin M, Ornek F. Long-term results of cataract surgery in patients with anterior uveitis. Int Ophthalmol. 2018;38(4):1399-407.

17. Mora P, Gonzales S, Ghirardini S, et al. Perioperative prophylaxis to prevent recurrence following cataract surgery in uveitic patients: a two-centre, prospective, randomized trial. Acta Ophthalmol. 2016;94(6):e390-4.

18. Ram J, Gupta A, Kumar S, Kaushik S, Gupta N, Severia S. Phacoemulsification with intraocular lens implantation in patients with uveitis. J Cataract Refract Surg. 2010;36(8):1283-8.

19. Razeghinejad MR, Katz LJ. Steroid-induced iatrogenic glaucoma. Ophthalmic Res. 2012;47(2): 66-80. 